\title{
Research on Effect of Culture Substrate Using Green Waste as Materials on the Growth of Herbaceous Flowers
}

\author{
Shuisheng $L u^{1, a}$ \\ ${ }^{1}$ Nanchang Institute of Science and Technology, 330108 \\ alushuisheng@126.com
}

Keywords: green waste; materials; growth of herbaceous flowers; research and analysis

\begin{abstract}
In modern society, increasingly more emphasis are put on landscape greening rehabilitation, which significantly improves our work and living environment and is good for our health. However with its amount increases steadily, green waste inflicts some damage on the ecological environment, and hinders the further improvement of landscape greening. To deal with the heaps of green waste better, we can recycle it as waste. However we can't dispose the green waste comprehensively and effectively with the present techniques, so this paper focuses on the analysis of the composting effect of culture substrate using green waste as materials on the growth of herbaceous flowers, and conducts research on its techniques and makes improvements.
\end{abstract}

\section{Introduction}

Green waste are the substances, comprising falling leaves, branches and flowers as well as weed and so on, that can be reused by soil ${ }^{[3]}$.However with the excessive requirement of environment beautification, we deal with the green waste in the direct and prompt method of incineration and landfill, both inflicting damage on the ecological environment. Furthermore, the incineration produces toxic substances released into the atmosphere, contaminating the air and endangering our health. To solve the problems, we can crush the green waste, and process it with a series of biological treatments to make culture substrate capable of improving the soil properties and maintaining water and soil balance. The method is beneficial to the further growth of the herbaceous flowers. Despite the numerous advantages of recycling green waste, we can't overlook its disadvantage, which is the need of adequate technological support to achieve the transforming project.So in view of the foregoing two points, I make further analysis and research to determine the most suitable and effective method of green waste processing to promote the growth of herbaceous flowers, thus improving the quality of our living environment.

\section{Research and analysis of culture substrate techniques}

The excellence of culture substrate,being one of the main methods to dispose green waste, has a direct influence on the growth of herbaceous flowers. The culture substrate with excellent qualities possesses adequate permeability, holding moisture and contains abundant nutrition to support the proper growth and development of vegetation. To achieve excellent cultivation, there are rigorous requirements on physical and chemical environments and the stability of bio system. As for culture substrate made through biological treatments, we can start analyzing it from soil. Biological treatments can alter the physical and chemical properties, and further improve the microbe content in thesoil, thus making the soil more fertile. Secondly, it can sustain the growth of various kinds of vegetation, and can be applied to the massive cultivation of herbaceous flowers in landscaping, thus achieving the better maintenance of the environment. In summary, I make analysis and research on the aspects of soil, microbe, physical and chemical properties and stability. However considering the fact that our country just starts primary research and techniques application of culture substrate made from green waste, there isn't an integrated system of research, manufacture and application. During the process we crush green waste, such as branches, leaves and weed, and mix the crushed substances with peat and garden soil in some specific ratio, to make the substrate for the growth of 
herbaceous flowers. But the culture substrate made through these simple techniques isn't stable enough to grow perfect herbaceous flowers. So fostering the best culture substrate through the strategy research and analysis targeting the factors resulting in the instabilities becomes the most urgent problem for researchers and enterprises.

\section{Factors influence the culture substrate}

\section{Physical and chemical factors}

As for culture substrateusing green waste as materials, the physical and chemical factors influencing the growth herbaceous flowers include: temperature, $\mathrm{PH}$ value, carbon-nitrogen-oxygen ratio and water moisture and so on. Among all the factors, temperature remains one of the most important factors that influence the microbe in the soil where herbaceous flowers grow. Rising temperature results in the rapid degradation and reproduction of the microbe existent in culture substrate, and prompts the growth of herbaceous flowers. However, overheat can affect the proper growth of vegetation, and might even cause the withering and death of herbaceous flowers. But if the temperature is too low, it can inhibit the development and reproduction of microbe, thus preventing the herbaceous flowers from flourishing healthily. $\mathrm{PH}$ value is the second physical factor affecting the culture substrate using green waste as materials, because some vegetation flourishes in sour environment, whereas others flourish in the alkaline environment. $\mathrm{PH}$ value of the culture substrate affects the growth of microbe andcomposting efficiency, so if the $\mathrm{PH}$ value of the environment is suitable for the growth and reproduction of microbe, it can form excellent culture substrate to sustain the health growth of herbaceous flowers. Carbon element, nitrogen element and oxygen element are important in determining the excellence of the culture substrate. Ventilation, oxygen supplying and supplementation of carbon and nitrogen energetic substance are crucial to culture substrate. Some gap should be maintained in the culture substrate for the drainage of its own water moisture and waste, as well as the air exchange with the outer environment, thus providing adequate oxygen for the microbe to survive in the culture substrate, which sustaining the proper growth of vegetation. However both redundant water moisture and materials accumulation can prevent the proper growth and reproduction of the microbe, thus bringing the growth of herbaceous flowers to a halt.Besides, sufficient carbonaceous energy can be used by the microbe surviving in the substrate to conduct energy conversion; whereas most nitrogen resources can't be used directly by microbe before being decomposed into other substances for the further usage. The ratio of carbon to nitrogen existing in the substrate directly affects the growth and development of microbe, and the feasible specific value of the ratio ranges from 26 to 34[4], being able to provide sufficient nutrition and energetic substances.Water, another essential element for microbe to survive in culture substrate, can lower temperature, dilute the density of carbon and nitrogen and slow the change of acid-base property to some degree. Insufficient water makes the culture substrate become dry, thus suppressing the metabolism of the existent microbe. However too much water might clog the gap in the culture substrate and renderthe existent microbe dead, resulting in putrefaction which is unfavorable for the further growth of vegetation. We can ensure the proper growth of herbaceous flowers by manipulating the stability of physical and chemical factors of culture substrate using green waste as materials. Table 1 shows Main physical and chemical properties of culture substrate.

Table 1 Main physical and chemical properties of culture substrate

\begin{tabular}{|llccc|}
\hline materialstotal & oxygen $\%$ & organic carbon $\%$ & water moisture $\%$ & C / N \\
\hline Green waste & 0.82 & 52.21 & 20.10 & 63.70 \\
\hline
\end{tabular}

\section{Techniques analysis of culture substrate using green waste as materials}

Speaking of the techniques, we start processing the substrate with chemical decomposing agent, which offsets the unfavorable effects brought by the lignin contained in green waste [1].In modern culture substrate techniques, the auxiliary chemical reagents play a significant role in landscape greening, because the chemical reactions between the reagents and chemical substances in the culture substrate produce energetic substances that can be absorbed by herbaceous flowers and 
other auxiliary substances favorable for the growth. During the formation of culture substrate, some physical and chemical factors aren't stable enough. For example, redundant water can clog the gaps in the substrate, and overheat or insufficient supply of oxygen can be unfavorable for the proper development of microbe, increasing the content of anaerobe which gives the culture substrate a rancid smell. So among the techniques employed by culture substrate, the containment of stink is applied to solve the problem. Currently the most frequently employed techniques is the application of physical absorbent, namely add chemical reagents to the substrate to contain the development of anaerobe. The theory of physical and chemical absorbent deodorization is to transform rancid substances into odorless substances, or change the density or structure of rancid ion in the air, so it makes air fresh and culture substrate where herbaceous flowers grow gives no rancid smell. When we finish making the culture substrate, we need to provide sufficient nutrition or ensure that the herbaceous flowers can synthesize nutrient substances for themselves before starting cultivating herbaceous flowers. The usual solution is doing some fertilization to supplement nutrition or kill theexistent pests. However culture substrate using green waste as materials can't absorb the fertilizer well enough, so it can't achieve the desired effect. The water-soluble fertilizer can be absorbed more quickly by herbaceous flowers, so it's an alternative solution. Whereas considering its pricey cost, water-soluble fertilizer isn't frequently applied by technical sections[1] . Analyzingthese techniques and applying them to the cultivation of culture substrate using green waste as materials can make up for some deficiencies in techniques of culture substrate.

\section{Analyzing the application of culture substrate techniques}

\section{Analyzing advantages of the application}

With the increasingawareness to environmental protection, there is more demand of green products, which we need to improve our living environment in the public space. We need all sorts of green products to absorb the sewage and exhaust gas, thus achieving the goal of water and air purification. Besides, we plant green products to improve our living quality. With the increasing amount of green products, it concerns us how to dispose the green waste. For example, the disposal of leaves, branches, flowers and fruits, produced in the trimming process of green products, is an urgent problem we have to solve in landscape greening.At present, scientists propose a concept that green waste is a part of green plants. Green waste comprises ofinorganic substance, such as nitrogen, phosphorus and potassium, and organic substance, whose content way surpasses those in the sludge. So the organic substance can be reused as fertilizer for the growth of herbaceous flowers. Besides, green waste, as a part of green plants, is rarely contaminated, so if we can turn green waste into organic fertilizer for the purpose of soil improvement, it will lower the cost of landscape greening and generate significant economic and social benefits.

\section{Analyzing disadvantages of the application}

Despite the significant benefits generated through the efficient disposal of green waste, we should never fail to notice the numeroustechnological problems existing in the process of its application. The biggest technological problem of green waste is that its structure is so complicated that it needs physical, chemical and biological methods to be decomposed, which makes the application of the techniques sophisticated. Besides, the degradationof green waste gives a rancid smell, which is the result of microbial fermentation. The further application of green waste may be hindered, if we fail to optimize the techniques of microbial fermentation. The low efficiency of green waste composting and fertilityconservation and the easy loss of nutrition remain the problems that must be solved in the future application of green waste.

\section{Developing trend of the application}

In the future, we should focus on three directions to use green waste in a more efficient way, thus making them favorable to the growth of herbaceous flowers. Firstly, intensify the application of biological techniques, and use the techniques of biological decomposition to solve the problem of green waste degradation. Nowadays people propose to use culture propagation to accelerate the degradation of green waste, which will be the research direction of the application of culture substrate using green waste as materials. Secondly, accelerate the development of composting and 
fertility conservation techniques. Nowadays people propose a technique of soilless culture, which in fact is the cultivation technique of substituting the substrate with the mixture of composting substrate, water-retaining agent and peat.If the technique is mature enough, it will allow us to max out the usage rate of green waste. Thirdly, refine the classification of green waste application. We should realize the fact that different herbaceous flowers require distinctive culture substrate, and if we apply green waste in accordance with the requirements of herbaceous flowersgrowth, we can increase the efficiency of green waste application and enhance its effect.

\section{Conclusion}

In summary, recycling green waste not only make us dispose and use resources properly[2], making the substances return back to nature, but also generate social benefits. But during the current process of waste disposal, the techniques aren't perfect or mature, so many enterprises and construction companies dispose the waste through direct landfill or incineration, both of which inflict numerous bad effects. Furthermore, this techniques brings about high cost, so many enterprises, with their mind fixed on more profits, refuse the application of recycling the renewable resources.To solve to the problem, our country should provide some talent and technology support, and can make a profit from investing in such programs, which has profound significance to both enterprises and the masses. Making enterprises pay much more attention to this techniques and recycling waste is favorable to the harmonious development of society and the protection of ecological environment.

\section{References}

[1] HU Dao-qi. Research on Herbaceous FlowersGrowthbased on Culture Substrate using Green Waste as Materials[J]. Engineering Technology, 2016, 12(24): 165.

[2] FU Jin. Effect Observation ofgarden waste compost used as floriculture substrate [J]. Beijing Agriculture, 2013(10).

[3] ZHANG Zheng-jiu, WANG Yao, LIU Yu-ning, etc. Reviews on Public ParticipatingWill to the Recycling of green waste[J]. China’s Collective Economy, 2014, 12(04): 122-123

[4] LIU Yan-zhen.Reviews on Carbonization of Green Waste and its Application Technique[J]. Heilongjiang Science and Technology Information, 2013(18). 